\title{
Simulating Complex Organ Interactions: Evaluation of a Soft Tissue Discrete Model
}

\author{
Maud Marchal, Emmanuel Promayon, and Jocelyne Troccaz \\ TIMC-GMCAO Laboratory, \\ Institut d'Ingénierie de l'Information de Santé, \\ Faculté de Médecine, \\ 38706 La Tronche Cedex, France
}

\begin{abstract}
Computer assisted procedures play a key role in the improvement of surgical operations. The current techniques in simulation potentially lead to more accuracy, more safety and more predictability in the surgical room. Despite the important number of algorithms proposed for interactively modelling deformable objects such as soft human tissues, very few methods have attempted to simulate complex anatomical configurations. In this paper, we present a new approach for soft tissue modelling whose novelty is to integrate the interactions between a given soft organ and its surrounding organs. The proposed discrete model is compared to finite element method in order to quantify its performance and physical realism. The model is applied to the simulation of the prostate-bladder set.
\end{abstract}

\section{Introduction}

The human body is a system of complex interactions between organs and tissues. These interactions are particularly intricate in the case of soft tissues. Actually soft tissues are undergoing deformations depending on their own physical characteristics and contiguous organs motions. Two main directions are taken to model human soft tissues: the biomechanical approach and the computational discrete approach [1].

The biomechanical approach is based on continuum mechanics. The most frequently used framework of this approach is the Finite Element Method (FEM) which offers the advantage of being based on a strong theoretical background. However researchers face two kinds of difficulties when applying this method to computer aided medical simulation: high computational cost and awkward construction and updating of complex anatomical environments where rigid, elastic and active structures are present and interact.

Computational discrete approaches, such as mass-spring networks, offer a simpler alternative to the construction of such complex structures and can be more easily modified to match changing structures in a particular simulation. While they are not computationally demanding, these approaches are difficult to control and assess.

For both methods, there are few examples for the integration of multiple dynamic interactions between soft organs and their environment. This study 
presents a new soft tissue modelling method based on a discrete approach that allows such interactions to take place. The method named Phymul is a volumetric evolution of a surface model developed by Promayon et al. 2. It allows to model the objects with interior nodes. In addition, the method can integrate the incompressibility constraint for each component of the environment. It has been validated by comparisons with a finite element method as a "gold standard" of soft tissue modelling.

Our medical aim is to model the prostate and the surrounding organs and to simulate deformations in real time. Prostate cancer is the second leading cause of cancer deaths in men in several countries. The prostate is therefore subject to an increasing number of diagnostic and therapeutic procedures that are evaluated by physicians for potential improvements in care. Modelling the interactions between the prostate and its environment may allow more accurate procedures to be developed and accepted by urologists.

The paper is structured as follows. At first, the model and its operation are described. Then, comparisons with well-proven soft tissue modelling methods are presented. Finally the modelling of the prostate and its environment is introduced.

\section{Phymul: A Volumetric Evolution for a Soft Tissue Modelling Method}

In this section, a volumetric version of Phymul is presented. Phymul is a discrete model based on computer graphics modelling. The new proposed approach allows to define objects not only with surfacic nodes as in the original Phymul 2 but with interior nodes. The first paragraph presents the geometrical description of models with Phymul. Then, dynamics are described and finally, as soft tissue modelling is one of the major interest in our application, the specific modelling of elastic components is detailled.

\subsection{Geometrical Description}

Phymul allows building separated objects with their own properties and choosing the interactions between them. The components of our model are all derived from a main basis: a set of nodes describing the geometry of the object to model. Each node has a position, a neighbourhood and different properties depending on what kind of component it belongs to. The nodes can be placed by the user either on the surface or inside the component. A mass is assigned to each node in order to generate forces and dynamics. Moreover the user can choose to model each component with a surfacic description (only nodes on the surface) or with a volumetric description (nodes are present inside the component), depending on their physical properties.

Living structures are governed by three main kinds of components: rigid components to model skeleton, deformable components to model soft tissues and active deformable components to model muscles. The latter inherit deformable components properties. Components with different properties can be 


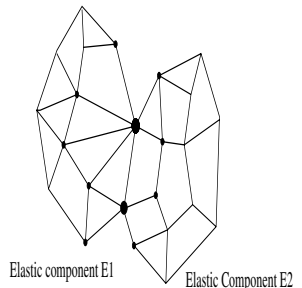

(a) Elastic-Elastic interaction

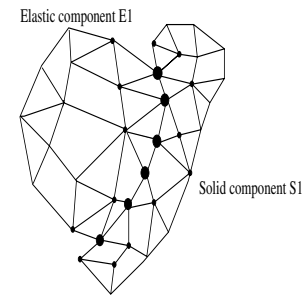

(b) Solid-Elastic interaction

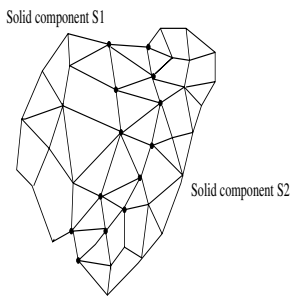

(c) Solid-Solid interaction

Fig. 1. Interaction configurations: (a) Border nodes (bigger black nodes) of elastic components belong to one component but have neighbours in the other elastic component. (b) Border nodes of solid and elastic components are doubled but constrained to be in the same position, whatever the forces applied on them. The forces on elastic component can thus be transmitted to solid component (for example, a link between a bone and a muscle). (c) Solid components are linked together by an elastic component (for example, two bones linked by a ligament).

easily stitched together in Phymul. Figure1 shows different configurations: interactions between two elastic components or two rigid components and interactions between an elastic and a rigid component.

\subsection{Modelling Description}

Besides the geometrical description, forces acting on each component and generating displacements and deformations have to be considered. Three kinds of forces can be used in the model: force fields (e.g. gravitation force), locally applied forces (e.g. forces generated by the user) and local shape memory forces to model deformable properties.

In addition to forces, constraints have to be implemented to model complex behaviours and to maintain some conditions such as non-penetrating volume. Phymul can deal with two kinds of constraints:

- local constraints that are applied to a single node, for example to keep a node in a particular region of space

- global constraints that are applied to a set of nodes, for example to enforce an object volume constraint.

The algorithm considers constraints as non-quantified force components: their satisfaction is guaranteed by using a direct projection algorithm based on the gradient vector of the constraint function. Position constraints can be easily defined at each node. For example volume preservation can be satisfied with a simple algorithm whose basis is detailled in [2]. In this method, nodes belonging to the surface are distinguished from nodes located inside the component to model. The algorithm works with all types of polyhedra: the mesh used for the geometrical description does not interfer in the volume computation. 
Finally, to solve the system dynamics, at each time $t$, the forces on each node are summed and the equations of motion are integrated taking into account the local and global constraints. The discrete integration scheme used for the equation of motion is Newton-Cotes scheme.

\subsection{Elastic Component}

Soft tissue modelling being our aim, let focus on the elastic component of Phymul, and detail how the elasticity property is built and computed. The elastic property is the ability for an object component to come back to its original shape once deformed, independently of the global object position. The elasticity in Phymul is described using an original formulation that has better stability properties than mass-spring networks, as shown by Promayon et al. 2. To model the elastic property of a node we define a local elasticity memory. The local shape memory force is generated on each node as follows: at each iteration, given the neighbour positions, we compute a shape ideal position: "an attraction node". Its position is ensured to locally minimize the deformation energy. When the node is at the shape nominal position, the local deformation and the shape memory force are both zero.

The difference between a mass-spring network and the local shape memory force is illustrated Fig. 2 with three nodes. Two nodes are fixed and a force is applied on the third node. With mass-spring system, the elasticity force is generated with two springs between the node and its neighbours. With the local shape memory, the elasticity force is represented by a spring between the node and its attraction node. The unique elasticity parameter of a given component is the stiffness of the spring on each node. Like mass-springs, local shape memory is easy to use for topological modifications.

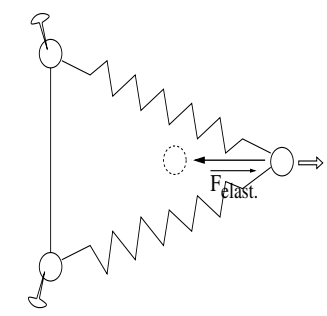

(a) Mass-spring system

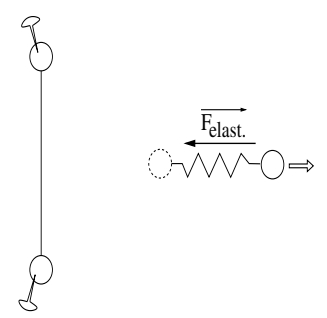

(b) Local shape memory

Fig. 2. Difference between mass-spring system and local memory shape

To compute the elastic property, a local shape coordinate system is defined on each node in order to find the shape ideal position whatever the object space orientation. Each node position is defined in the local coordinate system relatively to its neighbourhood at rest shape. During simulations, according to the neighbouring nodes position, a target position is computed for each node of an elastic component. 


\section{Comparisons with the Truth Cube Data and FEM}

New approaches of soft tissue modelling can be more easily experimented with phantoms than with complex tissue structures. The validation through these phantom studies are then considered to be applicable to various tissues using their own biomechanical properties.

In 2003 in a project named "Truth cube" 1, Kerdok et al. 3] validated their finite element deformation using the volumetric displacement data of 343 beads embedded in a silicone rubber cube and tracked by Computed Tomography. Material properties and geometry were known and boundary conditions had been carefully controlled while CT images were taken. Using this Truth cube data, we have been able to compare real data with both Phymul and the Finite Element Method.

A finite element model of the "Truth cube" has been built using commercial FE modelling software (ANSYS 8.0 software, Ansys Inc., Cannonsburg, PA). The material properties are isotropic and linear (with a Young Modulus of $14.9 \mathrm{kPa}$ and a Poisson ratio of 0.499 ). The cube edge dimension is $8 \mathrm{~cm}$ and the mesh is made of 8-node solid hexahedral linear elements, as in the model used by Kerdok. Large deformations are employed. For Phymul, the mesh was the same as the FEM mesh. The nodes are linked to form hexahedra. Comparisons have been achieved for three compression levels of $5 \%, 12.5 \%$ and $18.25 \%$. We focus on the distances between measured bead positions and those predicted by the two modelling methods. As measurement, we used the following formula with $d$ the Euclidian distance:

$$
\frac{d(\text { realBeads_position_afterCompression, simulatedBeads_position })}{d(\text { realBeads_position_init,realBeads_position_afterCompression })} \times 100
$$

to obtain a distance between real data and simulated date relative to the real beads displacement. The latest results are in mean:

- for the 5\% compression level (mean real displacement: $2.64 \mathrm{~mm})$ : $35.6 \%$ (Phymul) and $33.8 \%$ (Ansys),

- for the $12.5 \%$ compression level (mean real displacement: $6.12 \mathrm{~mm}$ ): $21.5 \%$ (Phymul) and 20.2\% (Ansys),

- for the $18.25 \%$ compression level (mean real displacement: $9.17 \mathrm{~mm}$ ): $16.9 \%$ (Phymul) and $16.8 \%$ (Ansys).

FEM produces lower mean errors than our model. Nevertheless, distances are very small compared to global characteristics. More precisely differences between FEM and our model are less than $0.2 \mathrm{~mm}$ for $5 \%$ compression. Concerning the computational cost, our method presents like other discrete approaches the advantage to be linear in the number of nodes and so enables the building of complex models with as necessary nodes as needed. On a Pentium $42.4 \mathrm{GHz}$, it takes $0.017 \mathrm{~ms}$ per node for each iteration. The number of iterations depends

\footnotetext{
${ }^{1}$ Data are available: http://www.medicalsim.org/truthcube/
} 
on the integration scheme. The FEM time results obtained with Ansys cannot be directly compared with Phymul's values, since a single step computational cost cannot be determined. Indeed, simulation time strongly depends on the hypothesis (non-linearity for example).

\section{Prostate Modelling in Its Environment}

After the validation with simple objects, more complex models containing several objects and better fitting to the prostate shape have been introduced. Modelling the prostate and its environment remains difficult because many factors are influencing prostate motion and deformation. Indeed, measurements of prostate position have demonstrated movements [4] and deformations [5] of the organ mainly due to rectum and bladder filling and recently due to breathing [6] and patient position.

In this paper, our aim is to simulate the influence of an endorectal echographic probe on prostate shape in function of bladder filling. The model presented contains three components: the prostate, the bladder and the rectum. An anatomic view of the prostate and its environment during a biopsy is represented in Fig. 3

The prostate and the bladder are represented by two deformed spheres. Bladder shape is deformed under gravity and is described with surfacic nodes (like an envelope). Prostate has a volumetric description. The echographic probe inside the rectum is simulated by the applied forces with a cylinder shape. Proportional scales of all organs are respected. Bladder and prostate are stitched together with a limited number of nodes. Top node of the bladder is fixed for each simulation with a different bladder shape (see Fig. 3 b) simulating different bladder repletions. During simulations, a pressure equivalent to real applied forces for biopsy is applied inside the rectum upward to deform the prostate.

After simulations with variations of bladder volume, it appears that the measured deformations correspond to those observed during surgical gestures. The prostate tends to deform more when the bladder is empty (10\% of its height) than when the bladder is full ( $5 \%$ of its height). The simulated deformations match to experiment measurements performed during a biopsy or a brachytherapy.

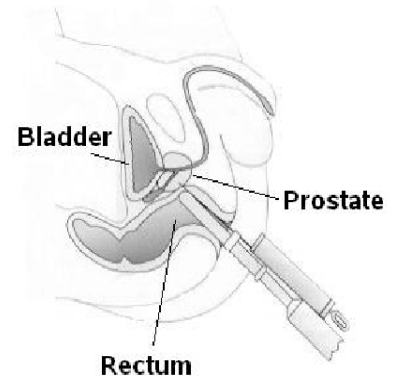

(a) Prostate anatomy

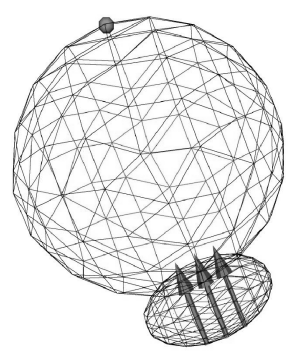

(b) Applied loads

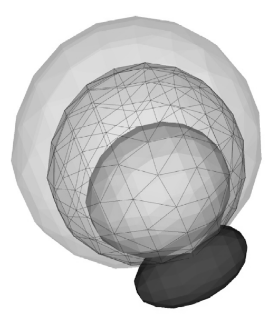

(c) Different bladder fillings

Fig. 3. Model of the prostate and different bladder fillings 


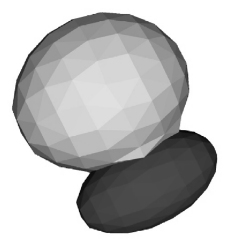

(a) Very small bladder

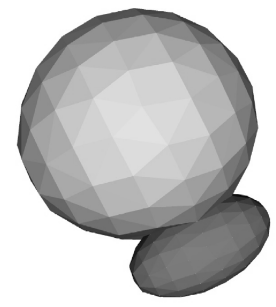

(b) Small bladder

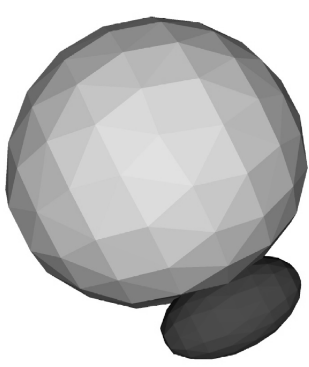

(c) Full bladder

Fig. 4. Prostate and bladder in different states

\section{Discussion and Conclusion}

In this paper, Phymul model and first simulations have been validated with objects of simple geometry. The first validation is a comparison with the Truth cube data and a finite element method. Results show that our method is relatively close to the FEM in terms of accuracy. A second set of experiments concerning the external shape of the cube has also been performed. Results show that our method performs similarly to the FEM in predicting deformations. It is important to note that there are a lot of other measurements to compare modelling methods. The distance choosen in this paper, although emphasis on a particular aspect, seems to us to be more relevant to compare both the discrete model and FEM to real data. The difference, even for FEM, shows us that simplistic modelling of object can induce relatively large difference in deformations, even for simple real object such as Truth cube. In a future work it could be of major interest to classify comparison measurements.

The second validation is directly linked to the prostate application. The model presented here is very simplified but allows to demonstrate how more complex models can be defined with simplicity with our method. We currently extend the work to a more realistic prostate and environment in terms of shape.

The incorporation of physical parameters in the model is a key element of soft tissue modelling. Even if it is possible to measure these parameters in isolated tissue [7, their determination is still very difficult to perform in vivo, especially since these parameters are patient and pathology specific. In addition the complexity of tissue interactions makes it difficult to perform a backward validation process. Prostate properties are reported in few papers [8]. Elastography has opened new possibilities to determine elasticity. Recently, Kemper et al. [9] published elasticity coefficients for the prostate. We have also performed another parallel study concerning the ability to translate FEM parameters (Young modulus for instance) in a local shape memory force. We plan to directly introduce Young modulus into Phymul through a force as a function of deformations. Future work will deal with modelling interactions with needles and ultrasound probe frequently used in biopsy or brachytherapy. 
Finally in this paper, a new approach to soft tissue modelling, well-suited to model interactions between organs has been proposed. Our object-oriented based method allows modelling several organs simultaneously. Contacts and interactions between tissues can be described more easily than in FEM for example. Compared to other discrete methods, we can define constraints like incompressibility and express objects properties with more accuracy and stability. By incorporating the interactions with instruments and by modelling more realistic organs, we will also be able to model the prostate behaviour by taking into account not only the organ itself but also its environment.

\section{References}

1. Delingette, H.: Towards realistic soft tissue modeling in medical simulation. IEEE Special Issue on Virtual and Augmented Reality in Medicine 86 (1998) 512-523

2. Promayon, E., Baconnier, P., Puech, C.: Physically-based model for simulating the human trunk respiration movements. In: Proceedings of CVRMed II - MRCAS III. (1997) 379-388

3. Kerdok, A., Cotin, S., Ottensmeyer, M., Galea, A., Howe, R., Dawson, S.: Truthcube : Establishing physical standards for real time soft tissue simulation. Medical Image Analysis 7 (2003) 283-291

4. Melian, E., Mageras, G., Fuks, Z., Leibel, S., Niehaus, A., Lorant, H., Zelefsky, M., Baldwin, B., Kutcher, G.: Variation in prostate position quantitation and implications for three-dimensional conformal treatement planning. Int. J. Radiation Oncology Biol. Phys. 38 (1997) 73-81

5. Forman, J., Mesina, C., He, T., Devi, S., Ben-Josef, E., Pelizarri, C., Vijayakumar, C., Chen, G.: Evaluation of changes in the location and shape of the prostate and rectum during a seven week course of conformal radiotherapy. Int. J. Radiation Oncology Biol. Phys. 27 (1993) 222

6. Malone, S., Crook, J., Kendal, W., Szanto, J.: Respiratory-induced prostate motion: quantification and characterization. Int. J. Radiation Oncology Biol. Phys. 48 (2000) 105-109

7. Fung, Y.C.: Mechanical Properties of Living Tissues. 2nd edn. Springer-Verlag (1993)

8. Krouskop, T., Wheeler, T., Kallel, F., Garra, B., Hall, T.: Elastic moduli of breast and prostate tissues under compression. Ultrasound Imaging 20 (1998) 260-278

9. Kemper, J., Sinkus, R., Lorenzen, J., Nolte-Ernsting, C., Stork, A., Adam, G.: Mr elastography of the prostate: initial in-vivo application. Rofo 176 (2004) 1094-1099 